\title{
Three-Band Simulation of the $g$-Factor of an Electron in an InAs Quantum Well in Strong Magnetic Fields
}

\author{
G. Gulyamov $\mathbb{D}^{1}{ }^{1}$ B. T. Abdulazizov $\mathbb{D}^{2},{ }^{2}$ and P. J. Baymatov $\mathbb{D}^{1}$ \\ ${ }^{1}$ Namangan State University, Namangan 160119, Uzbekistan \\ ${ }^{2}$ Physical-Technical Institute, Scientific Association "Physics-Sun”, Uzbekistan Academy of Sciences, Chingiz Aytmatov Street 2B, \\ 100084 Tashkent, Uzbekistan
}

Correspondence should be addressed to B. T. Abdulazizov; abdulazizovbahromjon@gmail.com

Received 23 February 2021; Revised 3 April 2021; Accepted 13 April 2021; Published 22 April 2021

Academic Editor: Filippo Giubileo

Copyright (c) 2021 G. Gulyamov et al. This is an open access article distributed under the Creative Commons Attribution License, which permits unrestricted use, distribution, and reproduction in any medium, provided the original work is properly cited.

The results of calculations of the Landau level and cyclotron $g$-electron factor in strong magnetic fields in an InAs quantum well based on both the three-band and two-band models are presented. The calculations were performed in the approximation of infinity of the depth of the quantum well, taking into account the Landau level of the second subband. It is shown that the three-band model satisfactorily describes the experimental data.

\section{Introduction}

Heterostructures (HS) based on the narrow-gap semiconductor InAs are promising for the creation of new-generation electronic and optoelectronic devices [1-3].

The cyclotron resonance (CR) experiment is widely used to study the band structure and spectrum of carriers in the quantum well of HS. In strong (quantizing) magnetic fields, this method makes it possible to determine the distance between the Landau levels (cyclotron energy) between which optical transitions occur and thereby obtain the cyclotron mass $[4,5]$. In weak magnetic fields, the effective carrier mass at the Fermi level can be determined. With the help of the $\mathrm{CR}$, one can reveal the nonparabolicity of the dispersion law and obtain information about the features of the dispersions of new materials $[6,7]$.

In work [7], an InAs QW based on the $\operatorname{InAs} / \operatorname{In}_{0.81-}$ $\mathrm{Ga}_{0.19} \mathrm{As} / \mathrm{In}_{x} \mathrm{Al}_{1-x} \mathrm{As}(x=0.52-0.81)$ structure was studied. The CR was used to measure the effective mass $m_{\mathrm{CR}}$ and $g_{\mathrm{CR}}$ electron factor at strong magnetic fields (up to $70 \mathrm{~T}$ ) and at different temperatures. The experimental results were interpreted qualitatively on the basis of the two-band Kane model and in the QW model of infinite depth; however, no quantitative comparison was made.
In strong magnetic fields and in wide QWs, the Landau levels of different subbands can approach or overlap sufficiently. Mathematical modelling of such processes using experimental values $m_{\mathrm{CR}}$ and $g_{\mathrm{CR}}$ makes it possible to better understand the band structure, the location of the Landau levels of the 1 st and 2 nd subbands, etc.

The aim of this work is to calculate the Landau levels of the 1 st and 2 nd subbands and, on the basis of these data, determine the dependence of the effective $g_{\mathrm{CR}}$ factor on the magnetic field. The calculations will be carried out on the basis of the three-band Kane model and the QW model of infinite depth. On the basis of these calculations, possible interpretations of the experimental results are discussed [7].

\section{Basic Formulas}

Consider the motion of one electron in the QW conduction band. Then, in the presence of a magnetic field applied perpendicular to the QW plane, the electron spectrum can be represented as $[4,8-10]$

$$
\varepsilon=\frac{m_{0}}{m^{*}(\varepsilon)}\left[\left(N+\frac{1}{2}\right) \hbar \omega_{0}+\frac{\hbar^{2} k_{z}^{2}}{2 m_{0}}\right]+\frac{g^{*}(\varepsilon)}{g_{0}} \sigma \hbar \omega_{0} .
$$


Here, $N$-Landau level numbers, $m_{0}$ and $g_{0}$, are the mass and spin splitting factor of a free electron, respectively, $\sigma$-electron spin $\pm 1 / 2$; $\hbar \omega_{0}$ denotes the cyclotron energy of a free electron

$$
\hbar \omega_{0}=\hbar \frac{e B}{m_{0} c}=1.16 \times 10^{-4} B\left[\frac{\mathrm{eV}}{\mathrm{T}}\right]
$$

In a conductivity band with nonparabolic dispersion, the band effective mass $m^{*}(\varepsilon)$ and the effective spin splitting factor $g^{*}(\varepsilon)$ of electrons are dependent on energy as [9-12]

$$
\begin{aligned}
\frac{m_{0}}{m^{*}(\varepsilon)} & =C+\frac{E_{\mathrm{P}}}{3}\left(\frac{2}{\varepsilon+\varepsilon_{\mathrm{g}}}+\frac{1}{\varepsilon+\varepsilon_{\mathrm{g}}+\Delta}\right), \\
\frac{g^{*}(\varepsilon)}{g_{0}} & =C^{\prime}-\frac{E_{\mathrm{P}}}{3}\left(\frac{1}{\varepsilon+\varepsilon_{\mathrm{g}}}-\frac{1}{\varepsilon+\varepsilon_{\mathrm{g}}+\Delta}\right) .
\end{aligned}
$$

Here, $E_{\mathrm{P}}$ is the Kane parameter, $\varepsilon_{\mathrm{g}}$ is band gap, and $\Delta$ is the magnitude of the spin-orbit splitting of the valence bands of a given semiconductor. Options $C, C^{\prime}$ characterize the effect of remote bands on the effective mass and effective $g$-factor of electron. Parameter value $C, C^{\prime}$ can be determined so that at the bottom of the conduction band $\varepsilon=0$, value $m^{*}(0)=m_{n}$ and $g^{*}(0)=g_{n}$ were equal to the observed value $m_{n}^{\exp t}, g_{n}^{\exp t}$. In the QW, the motion of an electron in the direction $z$ is also quantized. For example, in the QW model with an infinite potential barrier, $V=$ $\infty$, and width $L$ is the value of the wave vector $k_{Z}$ in formula (1), where is $k_{Z}=\pi n / L$ and $n$ is a spatial quantization level number.

When the split-off spin is sufficiently distant from the other branches, i.e., given that $\Delta>>\varepsilon_{\mathrm{g}}$ and besides, if $m_{n}<$ $<m_{0}$ from (1) and (3), a two-band model is obtained:

$$
\varepsilon\left(1+\frac{\varepsilon}{\varepsilon_{\mathrm{g}}}\right)=\frac{m_{0}}{m_{n}}\left[\left(N+\frac{1}{2}\right) \hbar \omega_{0}+\frac{\hbar^{2} k_{z}^{2}}{2 m_{0}}\right]+\frac{g_{n}}{g_{0}} \sigma \hbar \omega_{0} .
$$

Here, $m_{n}$ and $g_{n}$ are the effective mass and spin splitting factor at the bottom of the conduction band of a given semiconductor. Spectrum (4) can be written in the form (1) if we define

$$
\begin{aligned}
& m^{*}(\varepsilon)=\frac{m_{n}}{m_{0}}\left(1+\frac{\varepsilon}{\varepsilon_{\mathrm{g}}}\right), \\
& g^{*}(\varepsilon)=\frac{g_{n}}{g_{0}}\left(1+\frac{\varepsilon}{\varepsilon_{\mathrm{g}}}\right)^{-1} .
\end{aligned}
$$

According to (1) and (3) or (4) and (5), the electron spectrum depends on the magnitude of the magnetic field $B$, spatial quantization level numbers $n$, Landau level numbers $N$,
TABLE 1: Band parameters of InAs QW.

\begin{tabular}{lc}
\hline$E_{\mathrm{g}}(\mathrm{eV})$ & 0.42 \\
$\Delta(\mathrm{eV})$ & 0.38 \\
$m_{n}\left[m_{0}\right]$ & 0.023 \\
$g_{n}$ & -15 \\
$E_{\mathrm{p}}(\mathrm{eV})$ & 22.2 \\
\hline
\end{tabular}

and spin value $\sigma$. The cyclotron mass and $g$-factor are determined by the formulas

$$
\begin{aligned}
\frac{m_{0}}{m_{\mathrm{CR}}(B)} & =\frac{\varepsilon(n, N+1, \sigma)-\varepsilon(n, N, \sigma)}{\hbar \omega_{0}}, \\
\frac{g_{\mathrm{CR}}(B)}{g_{0}} & =\frac{\varepsilon(n, N, 1 / 2)-\varepsilon(n, N,-1 / 2)}{\hbar \omega_{0}} .
\end{aligned}
$$

For an electron, most semiconductor conditions $m_{n}<<$ $m_{0}$ are well done. In the InAs semiconductor, the approximation $\Delta>>\varepsilon_{\mathrm{g}}$ is rude since $\Delta=0.38 \mathrm{eV}, \varepsilon_{\mathrm{g}}=0.42 \mathrm{eV}$ (see Table 1). Figure 1 shows graphical comparisons of models (3) and (5) for InAs.

The graph shows that regardless of the roughness, the approximation $\Delta>>\varepsilon_{\mathrm{g}}$, a two-band model for calculating mass, is acceptable, but the calculated $g$-factors differ markedly. Below, we study the field dependence of the $g$-factor of the electron $g_{\mathrm{CR}}(B)$ determined by formula (6) based on both three-band and two-band models.

\section{Dependence of the Effective $g$-Factor of Electron on the Magnetic Field}

As can be seen from definition (6), to calculate the dependence $g_{\mathrm{CR}}(B)$, you need to know the Landau levels $\varepsilon(n, N$, $\sigma)$ which can be found from Equations (1), (3), (4), or (5). Therefore, first, we plot the field dependence of the Landau levels $\varepsilon(n, N, \sigma)$.

The band parameters of InAs used in the calculations are presented in Table 1. The calculation results for InAs QWs with the width $L=20 \mathrm{~nm}$ are shown in Figure 2, which are obtained from Equations (1) and (3), i.e., from the threeband model.

The graph shows that the main level with $n=1$ and $N=0$ approximately linearly dependent on the magnetic field $B$, and the upper lines differ markedly from the linear law, which is due to the nonparabolicity of the conduction band. Starting from about $B \sim 30 \mathrm{~T}$, the main Landau level of the second subband lies lower than the second Landau level of the first subband, i.e., $\varepsilon(2,0, \sigma)<\varepsilon(1,1, \sigma)$. Then, at high temperatures and/or at large values of the width of the Landau level [13] $(\sim \Gamma \sqrt{B})$, level $\varepsilon(2,0, \sigma)$ can be partially populated.

Figure 3 shows the results of calculating the absolute value of the effective $g$-factor $g_{\mathrm{CR}}(n, N)$ according to formula (6) based on the three-band model. 

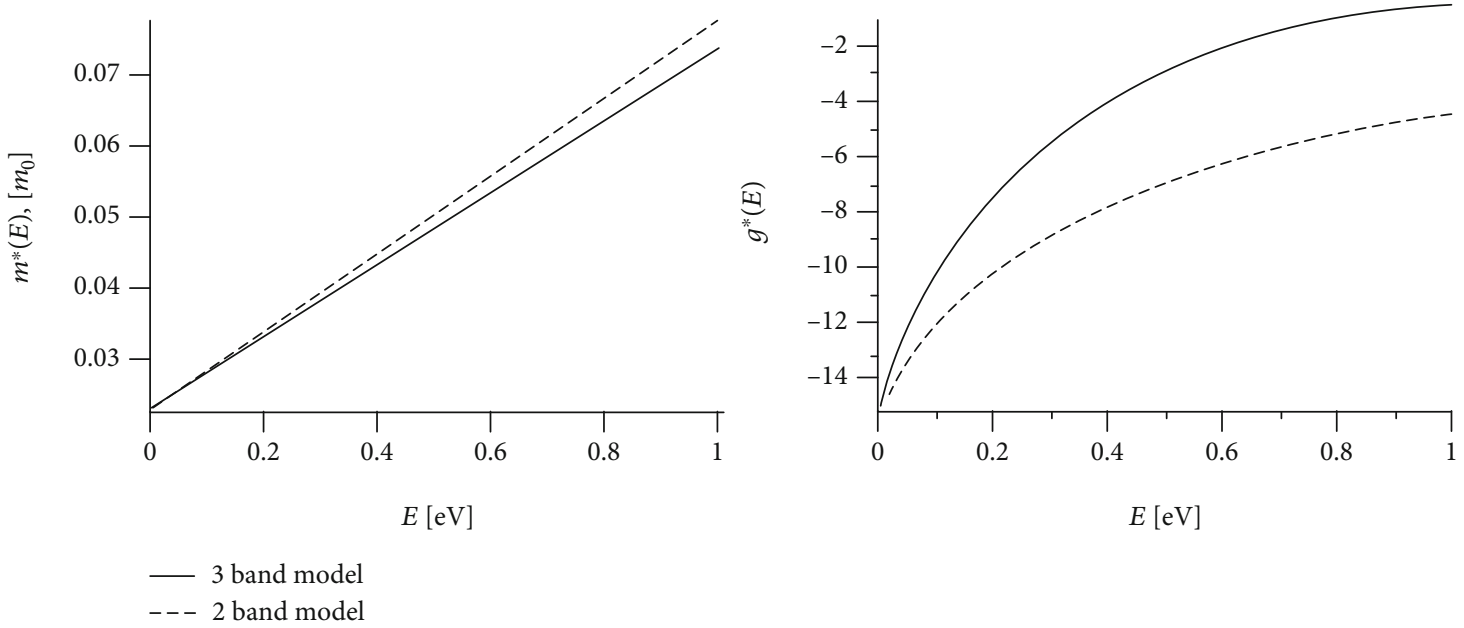

FIgURE 1: Comparison of the energy-dependent mass and electron $g$-factor for InAs determined by the three-band (3) and two-band (5) models.

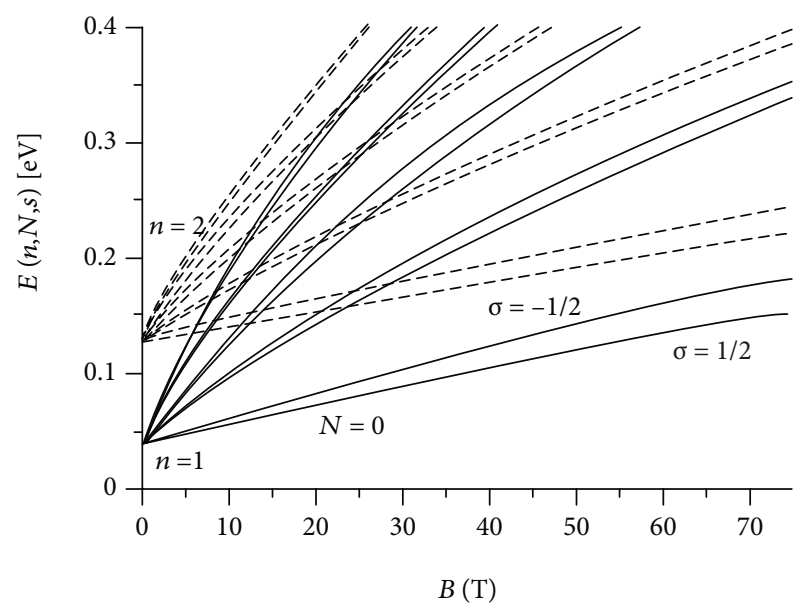

FIgURE 2: Landau levels of an electron in an InAs QW with $L=20$ $\mathrm{nm}$. Solid lines-levels for the first subbands; dashed lines-for the second subbands.

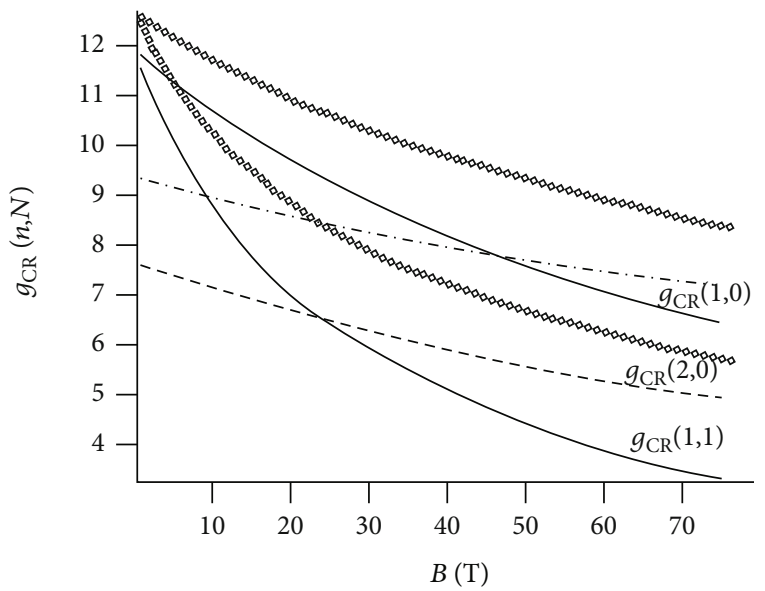

FIGURE 3: Dependencies $g_{\mathrm{CR}}(n, N)$ (absolute value) from magnetic field $B$ for an electron in an InAs QW with $L=20 \mathrm{~nm}$. Solid and dashed lines are obtained according to the three-band model, and dotted and dash-dotted lines according to the two-band model.

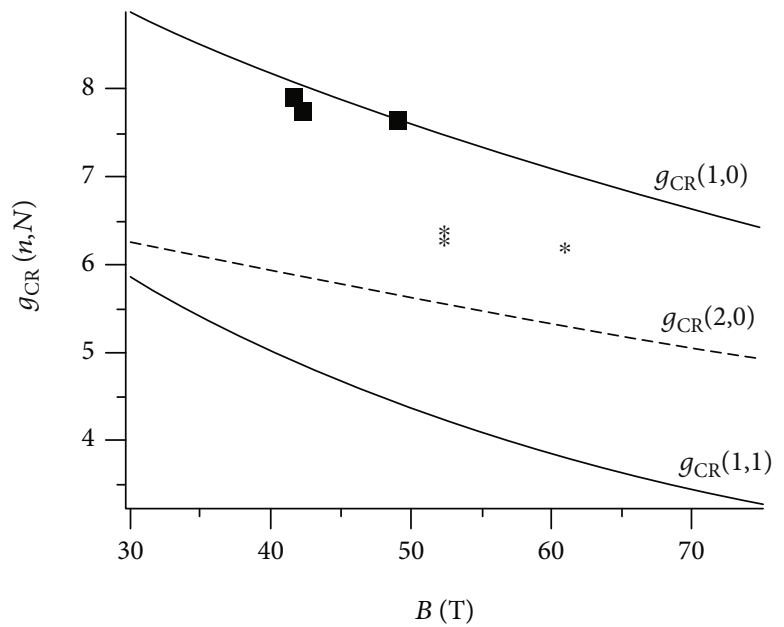

FIGURE 4: Comparison of absolute value dependence $g_{\mathrm{CR}}(n, N)$ from the magnetic field $B$ with experiment [7] for InAs QW with $L=20 \mathrm{~nm}$.

For comparison, the lines obtained on the basis of the two-band model are also shown. The graph shows that the results obtained from these models differ markedly; with increasing field $B$, this difference will increase.

Figure 4 shows comparisons of calculation results $g_{\mathrm{CR}}$ $(n, N)$ obtained from a three-band model with experimental data [7].

The graph shows that the measured first three points near $B \sim 45 \mathrm{~T}$ (they are indicated by squares (solid boxes)) agree satisfactorily with the theoretical line $g_{\mathrm{CR}}(1,0)$. Experimental points [7] $g_{\mathrm{CR}}(n, N)$ obtained at temperature $T=20.5 \mathrm{~K}$. Therefore, as can be seen from Figure 2, the Landau levels of the first subband lying above the Fermi level $E_{\mathrm{F}}$ cannot be partially populated. The main level of the second subband will be partially populated.

Experimental points at stronger fields $-B>50 \mathrm{~T}$ (they are marked with asterisks) lie roughly between the theoretical lines $g_{\mathrm{CR}}(1,0)$ and $g_{\mathrm{CR}}(2,0)$. These points can be referred to 
a line $g_{\mathrm{CR}}(2,0)$. The deviations can be associated with the approach of infinity of the QW depth, which noticeably overestimates the high-lying levels of spatial quantization. Note that the calculated line $g_{\mathrm{CR}}(1,0)$ obtained on the basis of the two-band model (this can be seen from Figure 3 ) lies significantly higher than these experimental points.

\section{Conclusions}

The value of the $g$-factor of an electron can be influenced by many factors, such as the finite height of the QW barriers, deformation effects, and structural asymmetry. Therefore, the calculations performed in this work are only a qualitative interpretation of the experimental results. However, some important facts follow from these results:

(i) Due to the noticeable difference, the dependences on the energy of the $g$-factor of the electron obtained on the basis of the two-band and three-band models (Figure 1), the calculated $g_{\mathrm{CR}}(n, N)$ lines of the cyclotron resonance also differ greatly (Figure 3 ). As can be seen from the graph in Figure 3 , in strong magnetic fields, the $g_{\mathrm{CR}}(1,0)$ line, obtained according to the three-band model, lies significantly lower than that given by the two-band model

(ii) In addition, when interpreting experimental data, taking into account the Landau levels of the second subbands is also important

The correct picture is restored when the model explains the concentration and field dependences of the Shubnikovde-Haas oscillations, optical transmissions, cyclotron mass, and $g$-factor measured in the experiment [7].

\section{Data Availability}

The data that support the findings of this study are available on request from the corresponding author (B. T. Abdulazizov).

\section{Conflicts of Interest}

The authors declare that they have no conflicts of interest.

\section{Acknowledgments}

The authors would like to thank Dr. Khimmatali Juraev for assisting during the proofreading of the manuscript. This work was financially supported by the Program for Fundamental Research Grant number BA-FA-F-2-005.

\section{References}

[1] B. R. Bennett, M. J. Yang, B. V. Shanabrook, J. B. Boos, and D. Park, "Modulation doping of InAs/AlSb quantum wells using remote InAs donor layers," Applied physics letters, vol. 72, no. 10, pp. 1193-1195, 1998.

[2] R. Magno, A. S. Bracker, and B. R. Bennett, "Resonant interband tunnel diodes with AlGaSb barriers," Journal of Applied Physics, vol. 89, no. 10, pp. 5791-5793, 2001.
[3] K. Ohtani and H. Ohno, "InAs/AlSb quantum cascade lasers operating at $10 \mu \mathrm{m}$," Applied physics letters, vol. 82, no. 7, pp. 1003-1005, 2003.

[4] I. M. Tsidilkovsky, Electrons and holes in semiconductors, Nauka, Moscow, Russia, 1972.

[5] M. J. Yang, R. J. Wagner, P. J. Lin-Chung et al., "Spin-resolved cyclotron resonance in a 2D electron gas," Surface science, vol. 305, no. 1-3, pp. 271-274, 1994.

[6] V. A. Kulbachinskii, N. A. Yuzeeva, G. B. Galiev et al., "Electron effective masses in an InGaAs quantum well with InAs and GaAs inserts," Semiconductor science and technology, vol. 27, no. 3, article 035021, pp. 1-5, 2012.

[7] J. Yuan, M. Hatefipour, B. A. Magill et al., "Experimental measurements of effective mass in near-surface InAs quantum wells," Physical Review B, vol. 101, no. 20, article 205310, 2020.

[8] M. Grundmann, "The Physics of Semiconductors," in An Introduction Including Devices and Nanophysics, p. 689, Springer-Verlag, Berlin Heidelberg, 2006.

[9] R. Winkler, Spin-Orbit Coupling Effects in Two-Dimensional Electron and Hole Systems, vol. 191, Springer Berlin Heidelberg, Berlin, Heidelberg, 2003.

[10] M. Roth, B. Lax, and S. Zwerdling, "Theory of optical magneto-absorption effects in semiconductors," Physics Review, vol. 114, no. 1, pp. 90-104, 1959.

[11] C. Weisbuch and C. Hermann, "Optical detection of conduction-electron spin resonance in GaAs,Ga1-xInxAs, andGa1-xAlxAs," Physical Review B, vol. 15, no. 2, pp. 816822, 1977.

[12] I. Vurgaftman, J. R. Meyer, and L. R. Ram-Mohan, "Band parameters for III-V compound semiconductors and their alloys," Journal of Applied Physics, vol. 89, no. 11, pp. 58155875, 2001.

[13] T. Ando, A. B. Fowler, and F. Stern, "Electronic properties of two-dimensional systems," Reviews of Modern Physics, vol. 54, no. 2, pp. 437-672, 1982. 